\title{
BMJ Open Demographic, clinical guideline criteria, Medicaid expansion and state of residency: a multilevel analysis of PrEP use on a large US sample
}

\author{
Pedro Botti Carneiro (D) ," Chloe Mirzayi (D) , , ${ }^{1,2}$ Scott Jones, ${ }^{3}$ Jonathon Rendina, ${ }^{3,4}$ \\ Christian Grov ${ }^{1}$
}

To cite: Carneiro PB, Mirzayi C, Jones S, et al. Demographic, clinical guideline criteria, Medicaid expansion and state of residency: a multilevel analysis of PrEP use on a large US sample. BMJ Open 2022;12:e055487. doi:10.1136/ bmjopen-2021-055487

- Prepublication history and additional supplemental material for this paper are available online. To view these files, please visit the journal online (http://dx.doi.org/10.1136/ bmjopen-2021-055487).

Received 15 July 2021 Accepted 17 January 2022

\section{Check for updates}

(c) Author(s) (or their employer(s)) 2022. Re-use permitted under CC BY-NC. No commercial re-use. See rights and permissions. Published by BMJ.

${ }^{1}$ Community Health and Health Policy, CUNY School of Public Health, New York, New York, USA ${ }^{2}$ Department of Epidemiology and Biostatistics, City University of New York, New York, New York, USA

${ }^{3}$ Whitman-Walker Clinic, Washington, District of Columbia, USA

${ }^{4}$ Department of Epidemiology, The George Washington University Milken Institute of Public Health, Washington, District of Columbia, USA

Correspondence to Dr Pedro Botti Carneiro; pedro.carneiro74@sphmail. cuny.edu

\section{ABSTRACT}

Objective To explore the association of clinical guidelinerelated variables, demographics and Medicaid expansion on pre-exposure prophylaxis (PrEP) uptake in one of the largest US sample of men who have sex with men(MSM) and transgender and gender non-binary (TGNB) people ever analysed.

Methods We cross-sectionally analysed predictors of current PrEP use using demographic and HIV risk-related variables (level-1), as well as state-level variables (level-2) (ie, Medicaid expansion status). We further explored the role state of residence plays in PrEP uptake disparities across the USA.

Results We found that the odds of PrEP use were significantly greater in older age, white, cisgender men. Moreover, individuals who reported recent post-exposure prophylaxis use, a recent sexually transmitted infection diagnosis and recent drug use were significantly more likely to report PrEP use. Finally, we found that the median odds of PrEP use between similar individuals from different states were 1.40 for the ones living in the Medicaid expansion states, compared with those not living in Medicaid expansion states. State of residence did not play a significant role in explaining PrEP disparities overall. Conclusion Our analysis showed that PrEP use is less common in communities standing to benefit the most from it-young MSM and TGNB of colour. However, individuals meeting federal guidelines for PrEP were significantly more likely to use PrEP. Though we found a positive association between living in Medicaid expansion states and PrEP use; that variable, as well as one's state of residency, were not suitable to explain variations in PrEP use in the US.

\section{INTRODUCTION}

In 2012, the US Food and Drug Administration (FDA) approved the first daily HIV preexposure prophylactic (PrEP) medication in the form of tenofovir disoproxil fumarate and emtricitabine. ${ }^{1}$ Following its approval, the Centers for Disease Control and Prevention (CDC) estimated that as many as 1.2 million Americans would benefit from taking the regimen. ${ }^{2}$ By 2017 , only approximately 80000
Strengths and limitations of this study

- This study reports on patient-level risk factors of pre-exposure prophylactic (PrEP) use in a sample of over 6000 cisgender men and transgender people who have sex with men across the USA, representing all states.

- This study uses multi-level modelling analysis to understand the role of state-level Medicaid-expansion alongside individual-level predictors on current PrEP use.

- This study includes the magnitude of clinical guidelines criteria predictors of PrEP use in a US national sample, and the role of Medicaid expansion on PrEP use.

- This study was conducted in 2017 and 2018, and the implementation of PrEP across the USA is ever growing and changing.

- The study uses self-reported cross-sectional data, and causal inference cannot be drawn from the analysis.

prescriptions were filled by unique HIVnegative users. ${ }^{3}$

Recent estimations of PrEP use in the general population suggest that as many as 200000 individuals have initiated or persisted in PrEP year-over-year since the 2012 FDA approval through 2020, ${ }^{4}$ a number still lower than expected. ${ }^{2}$ Researchers in the USA have reported PrEP discontinuation rates of up to $60 \%$ following 6 months of initiation. ${ }^{5-8} \mathrm{An}$ analysis of persistence (ie, continuous use) data using prescription drug records in the USA from 2012 to 2017 found that PrEP persistence was only 14 months on average, and significantly differed by race, age group and insurance status. ${ }^{9}$ Understanding this issue is critical for communities at-risk for HIV, especially black and Latinx men who have sex with men (MSM) communities. ${ }^{10}$ A limitation of current PrEP use reports in the USA is their reliance on pharmacy claims 
data, ${ }^{3}$ or multiple different sources to obtain estimates, ${ }^{4}$ limiting one's ability to account for confounding variables, like HIV-related risk factors. They also limit a deeper exploration of complex questions by limiting the study unit to a prescription claim, for example, rather than one individual. There is an immediate need to develop solutions to mitigate both issues-the overall uptake and persistence in PrEP, and the observed racial disparity in communities standing to benefit the most from it.

A prominent issue impacting PrEP uptake in the USA is coverage, both financial coverage in the form of health insurance, and geographic coverage in the form of access to a provider who is competent and accepts your medical coverage. Issues related to having health insurance coverage or being able to afford costs associated with medical care are widely reported throughout the PrEP literature, ${ }^{11-14}$ and they relate to an individual inability to pay for costs associated with taking PrEP. However, financial coverage is also managed at the state-level, through state-run Medicaid programmes and drug assistance programmes, which grant some access and affordability to PrEP. Patients enrolled in Medicaid have mixed levels of PrEP access, with enrollees with incomes under $150 \%$ of the federal poverty level (FPL) receiving PrEP for nearly free due to federal laws limiting costs. ${ }^{15}$ In 2010 , the Affordable Care Act (ACA) provided states with the ability to expand Medicaid programmes to adults aged 65 and younger with incomes 138\% of the FPL (about $\$ 17000$ a year) and below. ${ }^{15}$ Several states have chosen to expand Medicaid, and reports have linked increases in PrEP use to these expansions, ${ }^{16-18}$ suggesting that state variation in PrEP use may explain some of the disparities observed in the population at-large. Thus far, the issue of Medicaid expansion has only been explored on the aggregate level, limiting studies to measures of association which only informs on effect size but not on the impact of the variable on the distribution of PrEP across communities. Understanding the impact of Medicaid expansion on a population-level can support a better understanding of the complexities in regional disparities in PrEP use, for example, by exploring the association of Medicaid expansion and racial disparities in PrEP uptake. Furthermore, it seems imperative to understand whether the state of residency of a particular individual is significant to explain PrEP uptake.

Using the combined screening/enrolment data from two similar-in-scope US national cohorts, we created one of the largest national samples of MSM and transgender and gender non-binary (TGNB) individuals who have sex with men. Using a mixed-effect multilevel logistic analysis (MLA) approach, we analysed predictors of current PrEP use using demographic and HIV risk-related variables (level-1), as well as state-level variables (level-2) (ie, Medicaid expansion status).

\section{METHODS}

\section{About the studies}

The Together 5000 and UNITE studies are both US national cohorts longitudinally following sexual and gender minorities at-risk for HIV. Both cohorts are similar in scope, exploring sexual behaviour and PrEP uptake. Details on both studies have been described elsewhere. ${ }^{19}{ }^{20}$ Briefly, each used advertisements on geospatial sexual networking apps to recruit MSM and TGNB people who have sex with men across the USA to enrol in longitudinal assessments. During each study's enrolment phase, app-users were presented with an ad for the study. Those interested were directed to a brief screening (ie, eligibility) survey on their devices browser. The present analysis uses the reconciled screening data from each study dataset (ie, all variables that were identical across both screening surveys). Both studies enrolled samples in 2017 and 2018.

The sample's composition, which was not designed to be nationally representative, is nonetheless one of the largest national surveys of sexual minorities, consisting of 157035 responses, with $27 \%$ of the responses being from the Together 5000 study and the remainder from UNITE. Our current analysis, exploring individual-level and state-level predictors of current PrEP use, was limited to individuals not living with HIV, and those residing in one of the 50 states, Washington, DC or Puerto Rico-hereby referred to as 'states'. Our decision to limit the analytical sample to these states was based on state-level data availability. Our final sample was inclusive of 123905 (79\%) cisgender men and TGNB people who have sex with men.

\section{Individual-level variables (level-1) \\ Demographics}

Participants were grouped according to their age (under 24 years old, 25-29 years old, 30-49 years old, 50+ years old), gender identity (male, female (assigned male at birth), transgender person, something else) and race/ ethnicity (Black, Latino, white, multiracial, other).

\section{Current PrEP use}

Participants were asked about their PrEP status and current users were identified based on their self-reported status (current use/not).

\section{Clinical criteria guideline variables}

In both studies, participants were asked about postexposure prophylaxis (PEP) use in the prior 12 months, drug use in the past 3 months (ie, cannabis, cocaine, stimulants, methamphetamine, inhalants, sedatives, Gamma Hydroxybutyrate (GHB), ecstasy/MDMA, hallucinogen), and whether they received a sexually transmitted infection (STI) diagnosis (ie, syphilis, chlamydia or gonorrhoea) in the past 12 months. Based on their answers, we developed three dichotomous (yes/no) variables indicating their PEP, drug use and STI experiences within the timeframes noted. 


\section{State-level variables (level-2)}

\section{Medicaid expansion status}

We created a three-level variable to indicate the state's Medicaid expansion status as of 2020. We categorised as fully expanded, not expanded, or conditionally expanded. Conditional expansion includes any alternative Medicaid expansion model differing from the ACA format and one state that started expansion in 2020 (ie, Nebraska). ${ }^{21}$

\section{Analysis}

Our analysis included a descriptive assessment of our sample's demographics and HIV-clinical guidelinerelated variables, as well as a description of state-level variables. Next, we built a multilevel logistic multivariable mixed-effects regression model predicting current PrEP use (yes/no), using individual- (level-1) and statelevel (level-2) predictors. We calculated the fixed effects OR and 95\% CI of our fixed-effect variables, as well as the random effect intraclass correlation coefficients (ICC), the median ORs (MORs) of each of our models and the interval OR (IOR-80) of our fixed-effect level-2 variables-random effect components were calculated via previously reported equations and methods. ${ }^{22}{ }^{23}$ Our model-building approach was the following, first, we constructed a null model (model 1) in order to calculate the ICC and determine the variance in PrEP use accounted by an individual's state of residency. After, we built a model with level-1 variables (model 2) to explore the fixed effects of individual-level factors on current PrEP use. Finally, we built a full mixed effect multilevel logistic model (model 3) with all variables in both levels. Our analysis was conducted using the PROC GLIMMIX procedure with one random effect at the intercept, a binary distribution and a logit link on SAS V.9.4. We used Satterthwaite df. Random effects components were calculated manually. ${ }^{22}{ }^{23}$ Given our large sample size, we analysed our intervals of confidence and effect sizes when discussing statistical significance.

\section{RESULTS}

Our US sample varied demographically with over a quarter being under 24 years old, $13 \%$ were over 50 years of age, about $1.7 \%$ were transgender people and $40 \%$ were either bBack, Latino or multiracial. About $8 \%$ used PEP in the past 12 months, $60 \%$ used drugs in the past 3 months, and $13 \%$ had a positive STI results in the past 12 months. In total, $15 \%$ of the sample were current PrEP users, and the proportion of PrEP use was significantly greater in adults older than 29 years old ( $68 \%$ vs $51 \%$ ), white participants ( $59 \%$ vs $53 \%$ ), people who recently used PEP (23\% vs $5 \%$ ), who recently used drugs ( $74 \%$ vs $58 \%$ ) and those who reported a recent STI diagnosis (29\% vs $10 \%)$. Table 1 provides further details about our sample individual-level (level-1) variables. States-level characteristics (level-2) also varied greatly with about $54 \%$ having fully expanded Medicaid, and 22\% having conditionally expanded. We provided this list as an appendix (see online supplemental file 1).

Our regression model results are presented in table 2, we report here the findings of our model 3. The odds of current PrEP use for all age groups were significantly higher when compared with people 24 years old and younger, with individuals 25-29 having 2.2 greater odds (adjusted odds ratio $(\mathrm{aOR})=2.21,95 \% \mathrm{CI} 2.15$ to 2.28 ), 30-39 having 3.2 greater odds (aOR=3.20, 95\% CI 3.12 to 3.29 ) and those 50 years old and older having 2.9 greater odds (aOR=2.91, 95\% CI 2.82 to 3.01) of current PrEP use. All races had significantly lower odds current PrEP use when compared with white participants, with Black participants having $27 \%$ lower odds $(\mathrm{aOR}=0.73,95 \%$ CI 0.71 to 0.76$), 26 \%$ lower for Latino (aOR $=0.74,95 \%$ CI 0.73 to 0.76 ), and $21 \%$ lower for multiracial individuals (aOR $=0.79,95 \%$ CI 0.76 to 0.81 ). Those who identified as female $(\mathrm{aOR}=0.44,95 \%$ CI 0.35 to 0.56$)$ or as a transgender person $(\mathrm{aOR}=0.71,95 \% \mathrm{CI} 0.66$ to 0.77 ) had $66 \%$ and $29 \%$ significantly lower odds of being current PrEP users than those identifying as male. Individuals who reported PEP use in the past 12 months $(\mathrm{aOR}=3.94$, 95\% CI 3.85 to 4.04$)$, drug use in the past 3 months $(\mathrm{aOR}=1.73,95 \%$ CI 1.70 to 1.76$)$ or were diagnosed with an STI in the previous 12 months $(\mathrm{aOR}=3.34,95 \% \mathrm{CI}$ 3.27 to 3.42) had significantly greater odds of being current PrEP users. On the state level, individuals living in states with no Medicaid expansion had $31 \%$ lower odds of being current PrEP users (aOR=0.69, 95\% CI 0.54 to 0.88 ), and those living in conditional Medicaid expansion state had 27\% lower odds of being current PrEP users than individuals living in states with full expansion $(\mathrm{aOR}=0.73,95 \% \mathrm{CI} 0.56$ to 0.95$)$. For the states with no expansion $(\mathrm{aOR}=0.69)$ the IOR-80 was between 0.37 and 1.30 , and for those conditional expansions $(\mathrm{aOR}=0.73)$ it was between 0.39 and 1.38. The median odds of PrEP use between individuals with identical individual characteristics but from different states were 1.40 for the ones living in the Medicaid expansion states, compared with those not living in Medicaid expansion states. Overall, the state of residency accounted for about $6 \%$ in the variance of PrEP use overall, and after accounting for fixed-effects of individuals and Medicaid expansion, it accounts for only $4 \%$ of the remaining variance.

\section{DISCUSSION}

In this US national survey with over 120000 responses, we found that older age, white race, cisgender male identity and meeting objective criteria per current guidelines were positive predictors of current PrEP use. Previous epidemiological surveillance reports exploring PrEP uptake in the USA using prescription drug data have found similar demographic outcomes. ${ }^{3}$ Furthermore, though a state's Medicaid expansion status significantly predicted the likelihood an individual is currently taking PrEP, these effects were weak across states and did not explain variation in PrEP use in our analysis. Our study represents one 
Table 1 Demographics and clinical guideline-related characteristics of national sample of cisgender men and transgender people who have sex with men (level-1)

\begin{tabular}{|c|c|c|c|c|c|c|c|c|}
\hline \multirow[b]{2}{*}{ Variables } & \multirow{2}{*}{$\begin{array}{l}n \\
123905\end{array}$} & \multirow[t]{2}{*}{$\%$} & \multicolumn{2}{|c|}{ Not currently on PrEP } & \multicolumn{2}{|c|}{ Current PrEP user } & & \\
\hline & & & 104330 & $84 \%$ & 18126 & $15 \%$ & & \\
\hline Age group & & & & & & & 2711 & $<0.0001$ \\
\hline $25-29$ & 26347 & 21.3 & 22471 & 22 & 3876 & 21 & & \\
\hline $30-49$ & 48904 & 39.5 & 39370 & 38 & 9534 & 53 & & \\
\hline Gender identity ( $n=123453)$ & & & & & & & 69.81 & $<0.0001$ \\
\hline Male & 120861 & 97.9 & 102827 & 99 & 18034 & 99 & & \\
\hline Female (trans woman) & 388 & 0.3 & 367 & 0.4 & 21 & 0.1 & & \\
\hline Transgender person & 1959 & 1.6 & 1759 & 2 & 200 & 1.1 & & \\
\hline Something else & 245 & 0.2 & 225 & 0.2 & 20 & 0.1 & & \\
\hline White & 65941 & 53.2 & 55210 & 53 & 10731 & 59 & & \\
\hline Multiracial & 11845 & 9.6 & 10240 & 10 & 1605 & 9 & & \\
\hline Other & 7883 & 6.4 & 6709 & 6 & 1174 & 6 & & \\
\hline PEP in past 12 months $(n=123552)$ & & & & & & & 6616 & $<0.0001$ \\
\hline Yes & 9713 & 7.8 & 5542 & 5 & 4171 & 23 & & \\
\hline No & 113839 & 91.9 & 99717 & 96 & 14122 & 78 & & \\
\hline Drug use in past 3 months $(n=122456)$ & & & & & & & 1615 & $<0.0001$ \\
\hline Yes & 73837 & 59.6 & 60464 & 58 & 13373 & 74 & & \\
\hline No & 48619 & 39.2 & 43866 & 42 & 4753 & 26 & & \\
\hline
\end{tabular}

PEP, post-exposure prophylaxis; PrEP, pre-exposure prophylactic; STI, sexually transmitted infection.

of the largest US national samples to explore multilevel predictors of current PrEP use, using individual and riskrelated variables, and state-level variables.

Our findings affirmed the demographic disconnect between HIV and PrEP epidemiology in the USA. While HIV incidence is disproportionally distributed in black and brown MSM and TGNB youth communities, ${ }^{24} \mathrm{PrEP}$ was most commonly used by older white cisgender men. ${ }^{3}$ These effects persisted without change in effect size after controlling for Medicaid expansion. This contrast cannot be overlooked for the racial inequities in HIV outcomes in the USA are historic and enduring. Aside from denying protection to communities who stand to benefit the most from PrEP, demographic inequities in access to HIV prevention interventions can significantly increase the magnitude of this racial inequity. Nevertheless, PrEP use was much more common among those who would have otherwise benefited from its protection most, such as those who had taken PEP, been recently treated for an STI, or reported drug use. This scenario presents a critical consideration to the successes, and possible limitations, of current PrEP guidelines in the USA. ${ }^{25}$ The guidelines, set forth by the CDC, have a strong focus on objective risk (ie, recent bacterial STI, history of inconsistent or no condom use, sharing injection equipment). ${ }^{26}$ To that extent, our results demonstrated that guidelines can be successful in translating theory to practice: participants who reported any recent guideline criteria had as much as three times the odds of PrEP use than otherwise. However, the persistent demographic disconnect between who gets $H I V$ and who takes PrEP requires discussing the limitations of recommending PrEP solely based on objective risk. Researchers in the USA have previously speculated about the role an extension of guidelines would have in impacting PrEP uptake. ${ }^{27}$ Using the premise of determining 'good fit' of PrEP for a given patient's goals, instead of 'eligibility' for PrEP they suggest PrEP may be used to reduce HIV-related anxiety during sex and increase inter-partner intimacy. ${ }^{27}$ The CDC and other agencies overseeing clinical guidelines should immediately consider heeding such advice. Australia, for example, is considered a model-jurisdiction 
Table 2 Multilevel mixed-effects regression models predicting current pre-exposure prophylactic use

\begin{tabular}{|c|c|c|c|c|c|}
\hline Fixed-effects variables & Model 1 (null) & Model 2 & $95 \% \mathrm{Cl}$ & Model 3 & $95 \% \mathrm{Cl}$ \\
\hline \multicolumn{6}{|l|}{ Demographics } \\
\hline \multicolumn{6}{|l|}{ Age group (24 and under) } \\
\hline $30-49$ & & 3.20 & (3.04 to 3.37$)$ & 3.19 & (3.04 to 3.37 ) \\
\hline $50+$ & & 2.91 & (2.73 to 3.1$)$ & 2.9 & (2.82 to 3.01$)$ \\
\hline Latino & & 0.75 & (0.71 to 0.78$)$ & 0.74 & $(0.73$ to 0.76$)$ \\
\hline Multiracial & & 0.79 & (0.74 to 0.84$)$ & 0.79 & $(0.76$ to 0.81$)$ \\
\hline Other & & 0.82 & $(0.76$ to 0.88$)$ & 0.82 & (0.79 to 0.85$)$ \\
\hline \multicolumn{6}{|l|}{ Gender identity (male) } \\
\hline \multicolumn{6}{|l|}{ Risk variables } \\
\hline PEP use in past 12 months (ref: no) & & 3.94 & (3.76 to 4.14$)$ & 3.94 & (3.85 to 4.04$)$ \\
\hline STI diagnosis in past 12 months (ref: no) & & 3.34 & (3.21 to 3.48$)$ & 3.34 & (3.27 to 3.42$)$ \\
\hline Drug use in past 3 months (ref: no) & & 1.73 & (1.67 to 1.8$)$ & 1.73 & (1.7 to 1.76$)$ \\
\hline \multicolumn{6}{|l|}{ State-level variable } \\
\hline \multicolumn{6}{|l|}{ Medicaid expansion status } \\
\hline No expansion & & & & 0.69 & (0.54 to 0.88$)$ \\
\hline Conditional expansion & & & & 0.73 & (0.56 to 0.95$)$ \\
\hline \multicolumn{6}{|l|}{ Random effect components } \\
\hline Median OR (MOR) & 1.54 & 1.45 & & 1.40 & \\
\hline
\end{tabular}

PEP, post-exposure prophylaxis; STI, sexually transmitted infection.

for PrEP implementation, with several reports associating community PrEP uptake to substantial declines in HIV incidence. ${ }^{28} 29$ The guidelines for offering PrEP in Australia are much broader and comprehensive than those of the CDC, including reasons for offering PrEP such as "when a person plans to travel during which time they anticipate that they will be having condomless sex with casual partners', and 'when a person reports being so anxious about HIV infection that it may prevent them from having regular HIV testing, or engaging in any form of anal sex'. ${ }^{30}$ Thirty more inclusive set of clinical recommendations may have a much greater impact on PrEP uptake than traditional community outreach strategies; agencies and organisations with jurisdiction over these guidelines should consider doing so.

In exploring the role of a state's Medicaid expansion in predicting current PrEP use, we found mixed results. Online supplemental appendix 1 provides a detailed description of the state-level sample and some important demographic breakdown, as well as each state's Medicaid expansion status at the time of the study. The MOR $(=1.40)$ suggests that at least $50 \%$ of the odds of PrEP use between multiple pairs of identical individuals living in different states are $40 \%$ greater or higher, on average, for individuals living in states with Medicaid expansion. This finding is in line with previously reported effect estimates of PrEP use in relation to Medicaid expansion. ${ }^{16-18}$ However, our final model ICC indicated that the state of residency of a given participant accounted for only $4 \%$ of the variance of PrEP use in our analysis, and the IOR-80 for our Medicaid expansion variables measure of association (ie, OR) included the null value-1. In MLA, the inclusion of the null value on the IOR-80 indicates that the variable was not relevant to understanding the state-level variation in an individual predisposition to use PrEP. ${ }^{23}$ Furthermore, the positive MOR observed in 
our analysis must be understood in light of the small ICC presented in our model, though there may have been strong differences between two individuals from different states tendency to use PrEP, there was not enough variation between states for Medicaid expansion to impact PrEP use. In MLA, the estimate of the ICC is highly dependent on the area-level variable variance (eg, state size), ${ }^{22}$ which suggests that perhaps a smaller area-level analysis, like zip code or county-level, may be better suited to understand the impact of Medicaid expansion on PrEP uptake. Previously reported regional disparities in PrEP use seems to suggest this as well. For example, though Medicaid expansion has been associated with increased PrEP use, a majority of states have been found to have less than one PrEP-providing clinic per 100000 people. ${ }^{31} \mathrm{~A}$ narrower area of analysis, using MLA, may be advisable to explore how much geographic region explains disparities in PrEP use, and to explore the question about Medicaid expansion more effectively. The latter analysis, in fact, provides a better health equity framing to our question, because communities of colour often live in smaller enclaves, and using the entire state area may dissolve the true impact of the state's policy in these communities.

\section{Limitations}

Our findings must be understood in light of several limitations. First, our data were collected via self-report and may be subjected to social desirability bias. Several demographic variables that could further influence PrEP use were not measured such as health insurance status, income and other social determinants of health. Further, our outcome variable (current PrEP use) In our analysis we did not control from insurance type, for example, rather we explored the population-level effect of living in a Medicaid expansion state. It may be relevant to oversample patients receiving Medicaid and control for insurance information in future analysis.

The time our data was collected (2017-2018) is an additional limitation, and the relevance of the findings to the field of PrEP uptake might seem none. We call the reader's attention to the wholesome numbers of PrEP users reported in the USA-approximately 200 000-a stagnant number since then until now. ${ }^{8}$ We believe our findings provide some value to the question as to whether Medicaid expansion, as a variable, has an impact on an individual decision to start PrEP.

Finally, the parent studies of our dataset recruited participants using similar strategies that may have resulted in the same participants responding to both surveys. We note that we treated each individual response as independent. Although we cannot ascertain precisely the amount of overlap of participants across surveys, the studies' recruitment strategies used multiple applications platforms, each of which has millions of daily users. Therefore, the relative pool of available participants is several times the magnitude of those who actually took our surveys.

\section{CONCLUSION}

Our analysis showed that PrEP use is less common in communities standing to benefit the most from it-young MSM and TGNB of colour. However, individuals meeting federal guidelines for PrEP were significantly more likely to use PrEP. Updating guidelines may provide a strong avenue to improve uptake and reduce racial disparities. Additionally, individuals living in states where Medicaid was expanded were similarly more likely to use PrEP, however, we did not find that this variable was significant to explain state-level differences in PrEP use.

Acknowledgements We are thankful to participants for their time as well as other members of the Together 5000 study team. We gratefully acknowledge the contributions of all our participants within the UNITE study for their time and feedback. We would like to thank all the staff, students, and interns who made this study possible, with particular thanks to Ruben Jimenez, Brian Salfas, Dr Ali Talan and Nico Tavella. We are grateful for the time and contributions of Dr Mark Pandori and the Alameda County Public Health Laboratory. We would also like to thank collaborators, Drs Carlos Rodriguez-Díaz and Brian Mustanski. Data collection for this study was conducted at Hunter College of the City University of New York (CUNY), and affiliations reflect authors' institutions at the time of the most recent manuscript submission, which were not directly involved in the human subjects' portion of the research.

Contributors $\mathrm{PBC}$ wrote and was the primary lead on all aspects of the paper. PBC and CG conceptualised the paper, with PBC performing the main analysisboth reviewed multiple drafts and contributed to each section of the paper. CM performed the initial bivariate analysis, extracted the data, and provided valuable data analysis and variable development feedback. SJ Developed the dataset, conceptualised the variables, and reviewed multiple drafts of the manuscript. JR provided key methodology feedback and analytical guidance. Additionally, JR helped with the interpretation of results.

Funding This work was supported by the National Institutes for Health grant number UH3 Al 133675 (Grov) amd the National Institute on Mental Health, Eunice Kennedy Shriver National Institute on Child Health and Human Development, and National Institute on Drug Abuse (UG3/UH3-Al133674, PI: Rendina).

Competing interests None declared.

Patient consent for publication Consent obtained directly from patient(s)

Ethics approval This study involves human participants and was approved by Procedures for each of the cohort studies, as well as those to merge de-identified datasets were reviewed and approved by the CUNY Internal Review Board (Protocol number: 2019-0334). Participants gave informed consent to participate in the study before taking part.

Provenance and peer review Not commissioned; externally peer reviewed.

Data availability statement No data are available. This is secondary analysis.

Supplemental material This content has been supplied by the author(s). It has not been vetted by BMJ Publishing Group Limited (BMJ) and may not have been peer-reviewed. Any opinions or recommendations discussed are solely those of the author(s) and are not endorsed by BMJ. BMJ disclaims all liability and responsibility arising from any reliance placed on the content. Where the content includes any translated material, BMJ does not warrant the accuracy and reliability of the translations (including but not limited to local regulations, clinical guidelines, terminology, drug names and drug dosages), and is not responsible for any error and/or omissions arising from translation and adaptation or otherwise.

Open access This is an open access article distributed in accordance with the Creative Commons Attribution Non Commercial (CC BY-NC 4.0) license, which permits others to distribute, remix, adapt, build upon this work non-commercially, and license their derivative works on different terms, provided the original work is properly cited, appropriate credit is given, any changes made indicated, and the use is non-commercial. See: http://creativecommons.org/licenses/by-nc/4.0/.

ORCID iDs

Pedro Botti Carneiro http://orcid.org/0000-0002-4638-7824

Chloe Mirzayi http://orcid.org/0000-0002-9817-2868 


\section{REFERENCES}

1 Roehr B. FDA approves first drug to prevent HIV infection. BMJ 2012;345:e4879.

2 Smith DK, Van Handel M, Wolitski RJ, et al. Vital Signs: Estimated Percentages and Numbers of Adults with Indications for Preexposure Prophylaxis to Prevent HIV Acquisition--United States, 2015. MMWR Morb Mortal Wkly Rep 2015;64:1291-5.

3 Huang Y-LA, Zhu W, Smith DK, et al. HIV Preexposure Prophylaxis, by Race and Ethnicity - United States, 2014-2016. MMWR Morb Mortal Wkly Rep 2018;67:1147.

4 AVAC. PrEPWatch, 2020. Available: https://www.prepwatch.org/ country/united-states/

5 Marcus JL, Hurley LB, Hare CB, et al. Preexposure prophylaxis for HIV prevention in a large integrated health care system: adherence, renal safety, and discontinuation. J Acquir Immune Defic Syndr 2016;73:540-6.

6 Morgan E, Ryan DT, Newcomb ME, et al. High rate of discontinuation may diminish PreP coverage among young men who have sex with men. AIDS Behav 2018;22:3645-8.

7 Scott HM, Spinelli M, Vittinghoff E, et al. Racial/Ethnic and HIV risk category disparities in preexposure prophylaxis discontinuation among patients in publicly funded primary care clinics. AIDS 2019;33:2189-95.

8 Holloway IW, Dougherty R, Gildner J, et al. Brief report: PreP uptake, adherence, and discontinuation among California YMSM using Geosocial networking applications. J Acquir Immune Defic Syndr 2017;74:15-20.

9 Huang Y-LA, Tao G, Smith DK, et al. Persistence with human immunodeficiency virus pre-exposure prophylaxis in the United States, 2012-2017. Clin Infect Dis 2021;72:379-85.

10 Hess KL, Hu X, Lansky A, et al. Lifetime risk of a diagnosis of HIV infection in the United States. Ann Epidemiol 2017;27:238-43.

11 Mayer KH, Agwu A, Malebranche D. Barriers to the wider use of preexposure prophylaxis in the United States: a narrative review. Adv Ther 2020;37:1778-811.

12 Golub SA, Gamarel KE, Rendina HJ, et al. From efficacy to effectiveness: facilitators and barriers to PreP acceptability and motivations for adherence among MSM and transgender women in New York City. AIDS Patient Care STDS 2013;27:248-54.

13 Shrestha R, Karki P, Altice FL, et al. Measuring acceptability and preferences for implementation of pre-exposure prophylaxis (PreP) using conjoint analysis: an application to primary HIV prevention among high risk drug users. AIDS Behav 2018;22:2018-0401:1228-38.

14 Patel R, Singh S, Farag C, et al. Out-of-pocket costs impede PreP use among young MSM in the private healthcare system. Presented at Conference on Retroviruses and Opportunistic Infections, 2018.

15 Kay ES, Pinto RM. Is insurance a barrier to HIV preexposure prophylaxis? Clarifying the issue. Am J Public Health 2020;110:61-4.
16 Karletsos D, Stoecker C. Impact of Medicaid expansion on PreP utilization in the US: 2012-2018. AIDS Behav 2021;25:1103-11.

17 Patel RR, Mena L, Nunn A, et al. Impact of insurance coverage on utilization of pre-exposure prophylaxis for HIV prevention. PLoS One 2017;12:e0178737.

18 Baugher AR, Finlayson T, Lewis R, et al. Health care coverage and preexposure prophylaxis (PreP) use among men who have sex with men living in 22 us cities with vs without Medicaid expansion, 2017. Am J Public Health 2021;111:743-51.

19 Grov C, Westmoreland DA, Carneiro PB, et al. Recruiting vulnerable populations to participate in HIV prevention research: findings from the together 5000 cohort study. Ann Epidemiol 2019;35:4-11.

20 Rendina HJ, Talan AJ, Tavella NF, et al. Leveraging technology to blend large-scale epidemiologic surveillance with social and behavioral science methods: successes, challenges, and lessons learned implementing the unite longitudinal cohort study of HIV risk factors among sexual minority men in the United States. Am J Epidemiol 2021;190:681-95.

21 Foundation KF. Status of state action on the Medicaid expansion decision. Kff org, 2016.

22 Austin PC, Merlo J. Intermediate and advanced topics in multilevel logistic regression analysis. Stat Med 2017;36:3257-77.

23 Merlo J, Chaix B, Ohlsson $\mathrm{H}$, et al. A brief conceptual tutorial of multilevel analysis in social epidemiology: using measures of clustering in multilevel logistic regression to investigate contextual phenomena. J Epidemiol Community Health 2006;60:290-7.

24 CDC. HIV surveillance report, 2017. 29, 2018.

25 CDC. Preexposure prophylaxis for the prevention of HIV infection in the United States - 2017 update: a clinical practice guideline, 2018.

26 CDC. Preexposure prophylaxis for the prevention of HIV infection in the United States-2017 update: a clinical practice guideline. CDC website, 2018.

27 Golub SA, Myers JE. Next-Wave HIV pre-exposure prophylaxis implementation for gay and bisexual men. AIDS Patient Care STDS 2019;33:253-61.

28 Grulich AE, Guy R, Amin J, et al. Population-Level effectiveness of rapid, targeted, high-coverage roll-out of HIV pre-exposure prophylaxis in men who have sex with men: the EPIC-NSW prospective cohort study. Lancet HIV 2018;5:e629-37.

29 Medland NA, Grulich AE. HIV diagnoses in Australia fall as clinicians embrace pre-exposure prophylaxis. an independent review. 182, 2020.

30 Australasian Society of HIV VH, Medicine SH. Prep guidelines update: prevent HIV by prescribing PreP. Sydney: ASHM, 2019.

31 Siegler AJ, Bratcher A, Weiss KM, et al. Location location location: an exploration of disparities in access to publicly listed preexposure prophylaxis clinics in the United States. Ann Epidemiol 2018;28:858-64. 